\title{
Study on the interactions between transplanted bone marrow-derived mesenchymal stem cells and regulatory $T$ cells for the treatment of experimental colitis
}

\author{
DONGMEI ZUO ${ }^{1 *}$, XINGXING LIU $^{1 *}$, ZHEXING SHOU $^{1}$, HENG FAN $^{1}$, QING TANG $^{1}$, \\ XUEYUN DUAN ${ }^{2}$, DAN CAO $^{1}$, ZHOU ZOU $^{1}$ and LIJUAN ZHANG ${ }^{1}$ \\ ${ }^{1}$ Department of Integrated Traditional Chinese and Western Medicine, Union Hospital, \\ Tongji Medical College of Huazhong University of Science and Technology; \\ ${ }^{2}$ Hubei College of Traditional Chinese Medicine (TCM), Wuhan, Hubei, P.R. China
}

Received July 18, 2013; Accepted October 8, 2013

DOI: $10.3892 / \mathrm{ijmm} .2013 .1529$

\begin{abstract}
Accumulating evidence has established the use of mesenchymal stem cells (MSCs) as candidate cells for immunosuppressive therapy. Experimental studies have suggested that MSCs exert their immunomodulatory effects through the induction of regulatory $\mathrm{T}$ cells (Tregs) in vitro and in vivo. However, the interactions between MSCs and Tregs in inflammatory bowel disease (IBD) and whether MSCs can be used for the treatment of IBD remains to be elucidated. In this study, we aimed to investigate whether MSCs can be used for the treatment of IBD through the induction of Tregs. MSCs were isolated and identified by flow cytometry. The MSCs were transduced with a replication-defective recombinant lentiviral vector carrying GFP in order to be able to trace the injected cells in vivo. Prepared MSCs $\left(1 \times 10^{6}\right)$ were injected into rats with 2,4,6-trinitrobenzene sulfonic acid (TNBS)-induced colitis via the tail vein; the control rats received phosphatebuffered saline (PBS) alone. Two weeks after the intravenous infusion, the frequency of $\mathrm{CD} 4^{+} \mathrm{CD} 25^{+} \mathrm{Foxp} 3$ cells in the peripheral blood was examined by flow cytometry. The colon was sectioned and analyzed for histopathological changes. Foxp3 mRNA expression was determined by real-time reversetranscription polymerase chain reaction (qRT-PCR). In our study, the systemic infusion of MSCs significantly ameliorated
\end{abstract}

Correspondence to: Dr Zhexing Shou or Professor Heng Fan, Department of Integrated Traditional Chinese and Western Medicine, Union Hospital, Tongji Medical College of Huazhong University of Science and Technology, 1277 Jiefang Avenue, Wuhan, Hubei 430022, P.R. China

E-mail: 36747601@qq.com

E-mail: fanheng009@aliyun.com

${ }^{*}$ Contributed equally

Key words: bone marrow mesenchymal stem cells, regulatory $\mathrm{T}$ cells, inflammatory bowel diseases the clinical and histopathologic severity of TNBS-induced colitis in contrast to the controls. There was an inverse regulation of mucosal and peripheral Foxp3 expression, suggesting that the MSCs redistributed the Tregs from the mucosa to the blood. Thus, MSCs exhibit immunomodulatory functions and may be used to ameliorate or treat IBD by redistributing regulatory $\mathrm{T}$ cells. Therefore, the interactions between transplanted bone marrow-derived MSCs and Tregs should be further investigated; MSCs have tremendous potential for use in the treatment of IBD.

\section{Introduction}

Inflammatory bowel disease (IBD), which is a major gastrointestinal disease consisting of Crohn's disease (CD) and ulcerative colitis (UC), is a recurring and remitting disorder characterized by a range of symptoms, including abdominal pain, severe diarrhoea, rectal bleeding and wasting. It has been suggested that IBD results from environmental, genetic and immunological factors. Moreover, compelling data have suggested that the major contributing factor to IBD is an inappropriate reaction of the intestinal immune system to normal luminal enteric flora, according to individual genetic differences (1-3). CD and UC are distinguished by the tissues that are affected: $\mathrm{CD}$ can affect any region of the gastrointestinal tract in a discontinuous and transmural manner, whereas UC is restricted to the surface mucosa of the colon, in particular the rectum (4). Although the pathogenesis of these diseases is not yet fully understood, transmural inflammation in IBD, including lymphoid hyperplasia, submucosal edema, ulcerative lesions, as well as fibrosis is typically associated with the deficiency of T cell-mediated regulatory processes which would normally prevent and/or terminate inflammation (5). However, anti-inflammatory and immunosuppressive agents which we currently regard as important treatment regimens are not curative and only reduce the degree of intestinal inflammation associated with the disease (6). It is urgent to develop new therapeutic approaches that are more effective in the treatment of IBD.

Regulatory $\mathrm{T}$ cells (Tregs) are considered critical for maintaining immune homeostasis by establishing tolerance 
to foreign, non-pathogenic antigens that exist in commensal bacteria and food $(7,8)$. Patients who have non-functional or absent Tregs associated with genetic mutations in Foxp3 always have severe intestinal inflammation characterized by lymphocytic infiltration $(9,10)$. Similarly, mice lacking Foxp3 ${ }^{+}$Tregs are more likely to develop severe colitis (11). Previously, it has been suggested that the restoration of Tregs can prevent and treat colitis in mouse models of IBD (12).

Mesenchymal stem cells (MSCs) are multipotent cells which can present in the bone marrow and may be isolated from a variety of tissues, including adipose, liver, amniotic fluid, lung, skeletal muscle, as well as kidney tissue (13). On the basis of their convenient isolation, their low expression of human leukocyte antigen (HLA) and co-stimulatory molecules, as well as the fact that their isolation does not provoke ethical controversy, bone marrow-derived MSCs (BM-MSCs) are a promising alternative for cellular immunotherapy $(14,15)$. MSCs can not only differentiate into multiple cell lineages, including adipocytes, chondrocytes and osteocytes, but they also exhibit immunomodulatory functions in innate and adaptive immune systems. The immunosuppressive activity of MSCs is associated with a range of immune cells, including T, $\mathrm{B}$ and natural killer (NK) lymphocytes and dendritic cells (13). A number of in vitro and in vivo studies have suggested that MSCs exert their immunomodulatory effects through the expansion and/or induction of Tregs (16).

In view of their ability to differentiate into tissue-specific cell types, as well as their immunoregulatory effects in a variety of immune cell types, MSCs have the potential to prevent inflammatory disorders. A number of trials of MSC infusion for the treatment of graft-versus-host disease and $\mathrm{CD}$ have been published in recent years $(17,18)$. However, the interactions between MSCs and Tregs in IBD and whether MSCs can be used for the treatment of IBD remain unclear. In this study, we infused BM-MSCs into a 2,4,6-trinitrobenzene sulfonic acid (TNBS)-induced rat model of colitis in the effort to confirm our hypothesis that MSCs can be used for the treatment of IBD and to investigate the possible mechanisms behind the immunosuppressive activity of MSCs and Tregs.

\section{Materials and methods}

Animals. Female Sprague-Dawley (SD) rats of specific pathogen-free grade, weighing approximately 200 to $250 \mathrm{~g}$, were purchased from the Hubei Provincial Center for Disease Control, China. All rats were allowed free access to standard chow, as well as water and were randomly assigned to 3 different groups [control (untreated group), the TNBS + PBS group and the TNBS + MSC group]. The experimental protocol was approved by the Experimental Animal Center of Tongji Medical College, Huazhong University of Science and Technology, Wuhan, China.

Isolation and culture of BM-MSCs. The isolation and culture of the BM-MSCs was carried out as previously described (19). Four-week-old male rats were sacrificed by cervical dislocation. The rats were immersed in $75 \%$ ethanol for $5 \mathrm{~min}$, and the bone marrow was then isolated from the bilateral femurs and tibias. The cell suspension was then loaded onto a Percoll gradient (Sigma-Aldrich, St. Louis, MO, USA), and the cells were centrifugated for $5 \mathrm{~min}$ at $352 \mathrm{x} \mathrm{g}$. The top supernatant was then transferred into a tube and washed with phosphatebuffered saline (PBS). The cells were plated in a plastic tissue culture flask (Corning, NY, USA) and cultured in low-glucose complete cell culture medium consisting of $\alpha$-minimum essential medium ( $\alpha$-MEM; Gibco, Invitrogen Corp., Grand Island, NY, USA) containing $10 \%$ fetal bovine serum (FBS; HyClone Laboratories, Inc., Logan, UT, USA). The non-adherent cells were removed by adding fresh medium at $48 \mathrm{~h}$ and every 3-4 days thereafter. All the cultures were maintained at $37^{\circ} \mathrm{C}$ in a $5 \% \mathrm{CO}_{2}$ atmosphere. For approximately 1-2 weeks, only adherent cells were collected with $0.25 \%$ trypsin solution (Gibco, Invitrogen Corp.). The cells were passaged at approximately $80 \%$ confluency. Third-passage cells were used for all the experiments.

Cell-surface marker characterization of BM-MSCs. For flow cytometric analysis, the BM-MSCs were trypsinized and incubated for $30 \mathrm{~min}$ in the dark at $4^{\circ} \mathrm{C}$ with the following fluorescent antibodies: anti-CD29-PE-Cy7, anti-CD90-AlexaFluor ${ }^{\circledR} 488$, anti-CD45-PE and anti-CD11b-AlexaFluor ${ }^{\circledR} 647$ (BioLegend, San Diego, CA, USA). They were then washed twice with PBS, and the cells were resuspended in $300 \mu \mathrm{l}$ PBS. The detection of PE-Cy7/AlexaFluor 488/PE- and AlexaFluor 647-labeled cells was then carried out using a flow cytometer (FACSCalibur flow cytometer; Becton-Dickinson, Franklin Lakes, NJ, USA).

Transduction of BM-MSCs with GFP. Third-passage BM-MSCs at approximately $40 \%$ confluence were seeded in fibronectin-coated 6-well plates (Corning). The medium containing $10 \%$ FBS was removed, and the cells were then added to the replication-defective recombinant lentiviral vector carrying GFP (LV-GFP) supernatant containing $5 \mu \mathrm{g} / \mathrm{ml}$ polybrene (Shanghai Genechem Co., Ltd., Shanghai, China), to a final volume of $3 \mathrm{ml}$. The cells were transduced at a multiplicity of infection (MOI) of 20 units according to the manufacturer's instructions. Following incubation with LV-GFP for $2 \mathrm{~h}$, culture medium containing $10 \%$ FBS was added to the plates. An additional transduction was performed for $48 \mathrm{~h}$. After the cells were washed with PBS, the unabsorbed viral particles were removed. The BM-MSCs were then trypsinized for $5 \mathrm{~min}$ and used in the following experiments.

Induction of experimental colitis and treatment. Morris et al developed a simple and reproducible rat model of colonic inflammation in which they administered the hapten, TNBS (5-30 mg), in $0.25 \mathrm{ml}$ of $50 \%$ ethanol by intraluminal instillation. They also suggested that TNBS/ethanol-induced ulceration can persist for at least 8 weeks at a dose of $30 \mathrm{mg}$ (20). In our study, we used TNBS (Sigma-Aldrich) to induce experimental colitis according to the method mentioned above. Subsquently, on days 0,3 , and 7, the GFP-transduced BM-MSCs were injected via the tail vein at a dose of $1 \times 10^{6}$ cells in $0.3 \mathrm{ml}$ PBS into the rats with TNBS-induced colitis. In the control experiments, the animals received $0.3 \mathrm{ml}$ PBS without BM-MSCs according to the same protocol. Stool condition and body weight, as well as activity were evaluated daily to assess the disease activity index (DAI) as previously described (21). On day 15 , after the mice were sacrificed, blood was collected by ventral aorta puncture for the analysis of Tregs in peripheral 
blood and the entire colon was excised. The colon was divided for histological analysis and the evaluation of the expression of Foxp3 mRNA in intestinal mucosa.

Histological analysis. After the rats were sacrificed, the colon samples were fixed in $4 \%$ paraformaldehyde, embedded in paraffin and sliced into sections (4- $\mu \mathrm{m}$ thick) before staining with hematoxylin and eosin. Histological evaluation was completed by two expert pathologists in a blinded manner according to a scoring scale described previously (22). The evaluated parameters were as follows: damage/necrosis, inflammatory cell infiltration, submucosal edema, as well as hemorrhage of the mucosa. Each of the parameters estimated was graded as follows: 0 , no change; 1 , mild; 2 , moderate; 3 , severe changes. The histological score was defined as the sum of these parameters.

Tracing of GFP-labeled BM-MSCs. To confirm the location of the infused BM-MSCs in the inflamed colonic tissues, we transduced the BM-MSCs with LV-GFP. Colon tissue samples excised from the colon of the inflamed region and the non-inflamed region were embedded in optimum cutting temperature (OCT) compound (Sakura Finetechnical Co., Ltd., Tokyo, Japan), and then frozen on dry ice. One section was used to detected GFP-positive cells by fluorescence confocal microscopy (E600; Nikon, Tokyo, Japan) and the other was stained with an antibody against GFP (Millipore, Billerica, MA, USA) and visualized using FITC-conjugated secondary antibody (Santa Cruz Biotechnology, Inc., Santa Cruz, CA, USA). Other sections were also prepared to analyze GFP protein by western blot analysis. Total protein was isolated and quantified using a BCA protein assay kit (Pierce, Perbio Science, Tattenhall, UK). The primary antibody against GFP was used at a dilution of 1:1,000. For the control protein loading, anti-GAPDH was used at a dilution of 1:10,000. The protein extract was resolved by SDS-PAGE electrophoresis and transferred onto polyvinylidene difluoride (PVDF) membranes, which were blocked in 5\% BSA and incubated with the relevant antibody.

Analysis of Tregs in peripheral blood by flow cytometry. Peripheral blood mononuclear cells $\left(2 \times 10^{6}\right.$ cells $\left./ \mathrm{ml}\right)$ were stained at $4^{\circ} \mathrm{C}$ for $30 \mathrm{~min}$ with anti-CD4-FITC and anti-CD25-APC (all from Beckman Coulter, Brea, CA, USA). Following incubation, the cells were fixed with Foxp3 fixation/permeabilization buffer $1 \mathrm{ml}$ and permeabilized with Foxp3 permeabilization buffer $1 \mathrm{ml}$ to determine intracellular Foxp3 levels. The cells were then incubated for $30 \mathrm{~min}$ at $4^{\circ} \mathrm{C}$ with anti-Foxp3-PE antibody or isotype control (IgG2-FITC) (all from Beckman Coulter). The stained cells were then analyzed by flow cytometry.

mRNA expression of Foxp3 in the colon. The colon segments were frozen in liquid nitrogen until use at $-80^{\circ} \mathrm{C}$. We used TRIzol reagent (Invitrogen Corp.) to isolate the total RNA from the individual colons according to the manufacturer's instructions. cDNA was synthesized from $0.5 \mu \mathrm{g}$ of total RNA using a reverse transcription kit (Toyobo Co., Osaka, Japan) according to the manufacturer's instructions. Subsequently, a real-time PCR reaction with final volume of $20 \mu \mathrm{l}$ using the
SYBR-Green Realtime PCR master mix (Takara Bio, Inc., Shiga, Japan) was carried out using the ABI PRISM 7900HT sequence detector system (Applied Biosystems, Life Technologies, Carlsbad, CA, USA). Primers were designed according to the data from GenBank and evaluated by nucleotide BLAST standard search to avoid cross-reactivity with other known sequences. The designed primer sequences used for real-time PCR were as follows: Foxp3 upstream, 5'-GGCAAACGGAGTCTGCAAG-3' and downstream, 5'-TGCTCCAGAGACTGCACCAC-3'. The relative gene expression level, including the levels of the target gene, normalized to the endogenous and control gene was calculated using the comparative $\mathrm{Ct}$ method $2^{-\Delta \Delta \mathrm{Ct}}$.

Statistical analysis. Data are expressed as the means \pm standard deviation. Significant differences were assessed by the Statistical Package for the Social Sciences (SPSS) version 18 and Microsoft EXCEL version 2003. A p-value $<0.05$ was considered to indicate a statistically significant difference. We use an unpaired t-test and the Mann-Whitney $U$ test for parametric and non-parametric analyses between 2 groups.

\section{Results}

Characterization of BM-MSCs. At passage 3 or 4, the MSCs appeared to be a homogenous population of spindle-shaped cells, such as fibroblasts morphologically after the non-adherent cells were removed. Flow cytometric analysis confirmed that the BM-MSCs were positive for CD29 and CD90, but stained negative for the hematopoietic surface markers, CD45 and CD11b (Fig. 1).

General conditions. All the TNBS-treated rats developed clinical symptoms similar to IBD in humans 2-3 days after modeling, including looser stool, bloody purulent stool, and loss of weight and their activity significantly decreased. However, the rats that were not administered TNBS did not show any of the abovementioned signs and gained weight over time. Following the infusion of the BM-MSCs, the symptoms of looser stool and bloody purulent stool were significantly alleviated compared with the control group (Fig. 2).

Histological improvement in the colon following infusion of BM-MSCs. Microscopically, histological changes in the colon samples from the rats with TNBS-induced colitis were observed. In comparison to the control group, the rats in the MSC-treated group showed a relatively intact structure of colonic mucosa which consisted of more organized mucosal glands and more abundant goblet cells, milder congestion and edema and less inflammatory cell infiltration in the mucosa and submucosa (Fig. 3). The histological colitis score defined as the sum of those parameters was significantly reduced in the MSC-treated group (Fig. 4)

Localization of infused BM-MSCs in the colon. In vivo, we transduced the BM-MSCs with GFP to cofirm the localization of the infused BM-MSCs in the inflamed colonic tissues. In vitro, a large proportion of GFP-labeled BM-MSCs were observed (Fig. 5). However, we did not detect GFP fluorescence after 14 days under a confocal microscope in vivo. 

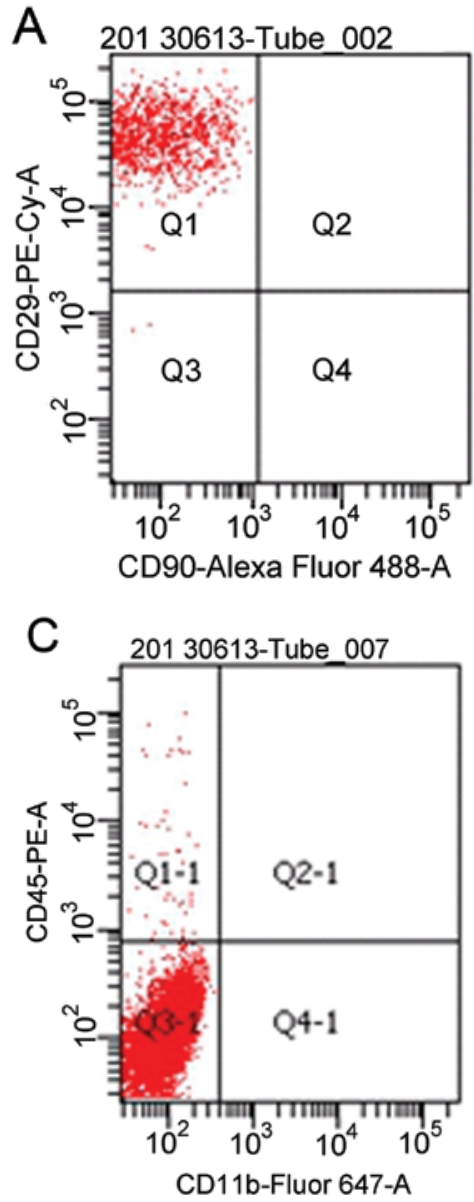
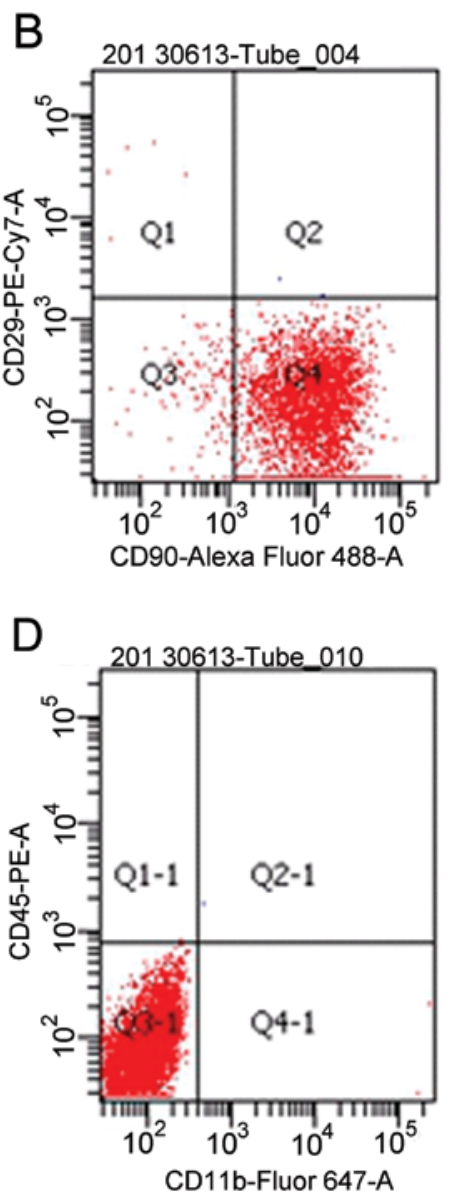

Figure 1. Mesenchymal stem cell (MSC) cell-surface antigen analysis. Bone marrow-derived MSCs (BM-MSCs) were positive for CD29 and CD90, but negative for CD45 and CD11b.

Distinct GFP-positive cells were observed when we used a GFP antibody and FITC-conjugated secondary antibody as described above, and the number of positive cells was then observed (Fig. 5). Moreover, high levels of expression of GFP protein were observed in the GFP-labeled BM-MSC group (Fig. 6). By contrast, no immunoreactivity was detected in the PBS-treated group and the normal control.

Effect of BM-MSC therapy on Tregs in peripheral blood. A number of studies on animal models of IBD have suggested that this disease is typically associated with a defect in the number of Tregs and that the restoration of Tregs can prevent and treat colitis $(23,24)$. For this reason, in this study, we evaluated the effects of infused BM-MSCs on Tregs in the peripheral blood in the rats with TNBS-induced colitis. The proportion of $\mathrm{CD} 4^{+} \mathrm{CD} 25^{+} \mathrm{Foxp} 3$ cells markedly decreased in the PBS-treated rats with TNBS-induced colitis compared with the control group $(\mathrm{p}<0.05)$, as shown by flow cytometry (Fig. 7). Following the infusion of BM-MSCs, the proportion of $\mathrm{CD} 4{ }^{+} \mathrm{CD} 25^{+} \mathrm{Foxp} 3$ cells incresed compared with the PBS-treated group $(\mathrm{p}<0.05)$ (Fig. 7); however, no difference was observed between the BM-MSC-treated group and the control (untreated) group.

mRNA expression of Foxp3 in colonic mucosa. The colonic mucosal mRNA expression of Foxp3 was determined in the

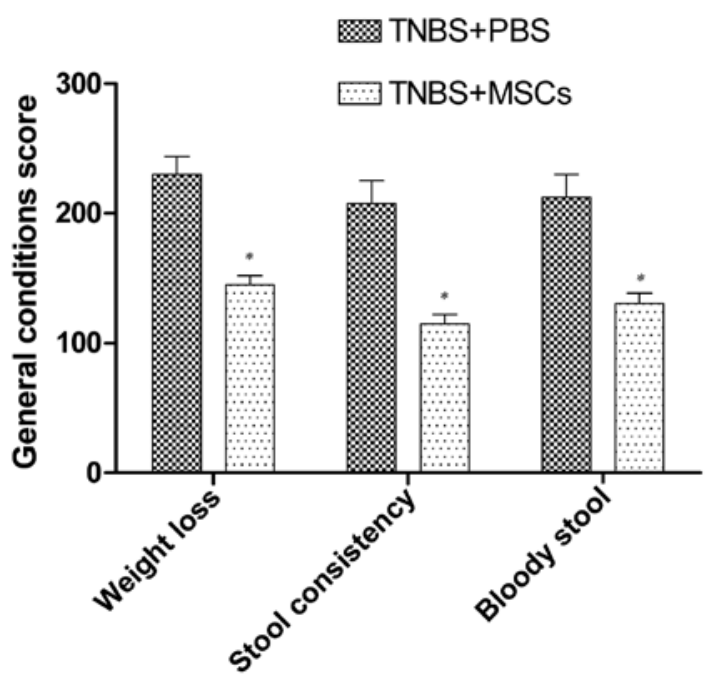

Figure 2. Clinical therapeutic efficacy of the transplantation of bone marrowderived mesenchymal stem cells (BM-MSCs) on the general conditions, such as body weight, stool consistency and rectal bleeding in rats with 2,4,6-trinitrobenzenesulfonic acid (TNBS)-induced colitis. Data are represented as the means \pm SEM ( $n=6$ rats per group, results are from 3 separate experiments). *p $<0.05$ vs. phosphate-buffered saline (PBS)-treated group.

BM-MSC-treated rats with TNBS-induced colitis and the PBS-treated group by real-time PCR. As shown in Fig. 8, an increase in the mRNA expression of Foxp3 was observed in 
A

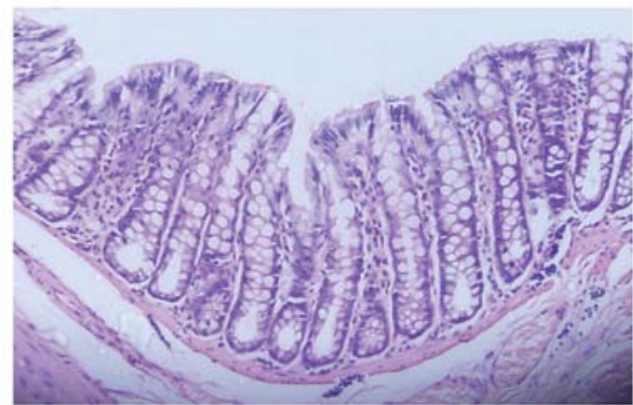

B

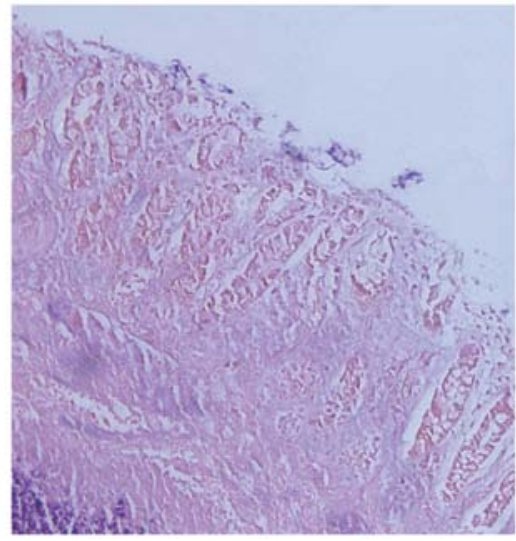

C

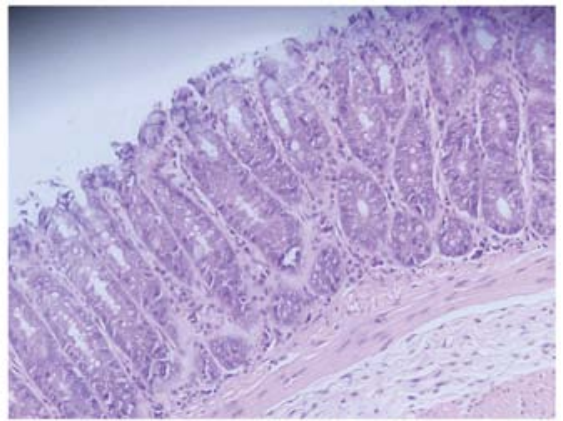

Figure 3. Histologic analysis after the infusion of bone marrow-derived mesenchymal stem cells (BM-MSCs) into rats with 2,4,6-trinitrobenzenesulfonic acid (TNBS)-induced colitis. Colon specimens were sectioned and stained with hematoxylin and eosin (H\&E). (A) Control group (magnification, $\mathrm{x} 20$ ); (B) phosphate-buffered saline (PBS)-treated group (magnification, $\mathrm{x} 20$ ); (C) BM-MSC-treated group (magnification, $\mathrm{x} 20$ ).

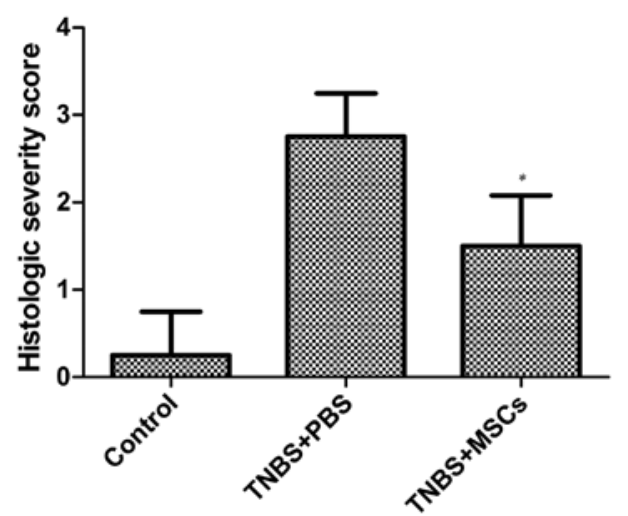

Figure 4. Effect of infusion of bone marrow-derived mesenchymal stem cells (BM-MSCs) on histological colitis score. BM-MSC-treated rats showed a significant lower histological severity score consisting of damage/necrosis, inflammatory cell infiltration, submucosal edema, as well as hemorrhage of mucosa compared with phosphate-buffered saline (PBS)-treated and control groups. Values are the means \pm SEM $(n=6$ mice per group, results are from 3 separate experiments). ${ }^{*}<0.05$ vs. PBS-treated group and control group.
A

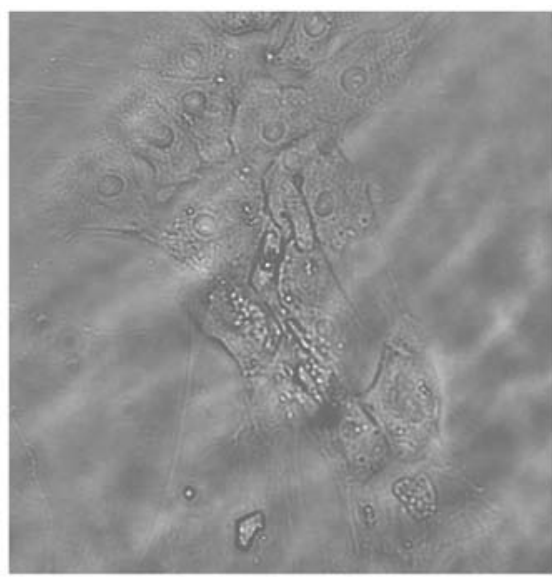

B

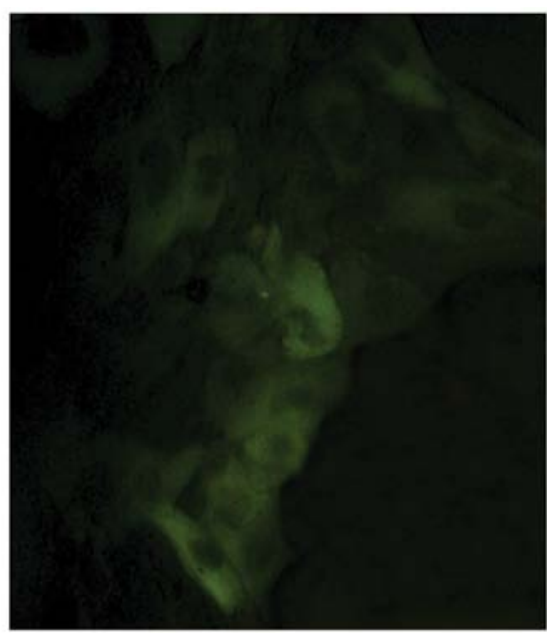

C

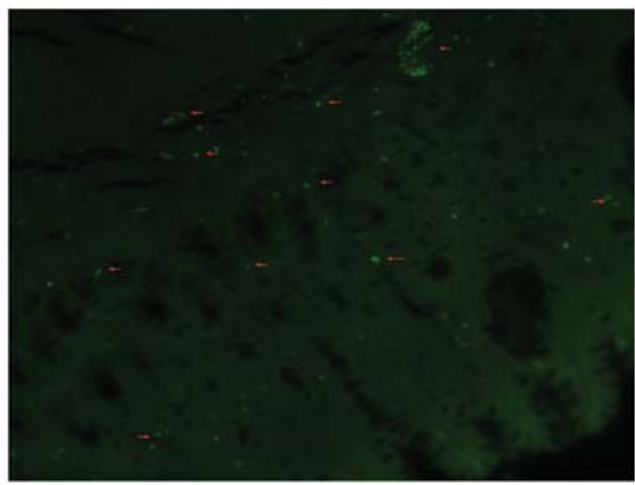

Figure 5. Localization of exogenously administered bone marrow-derived mesenchymal stem cells (BM-MSCs). (A) Recombinant replication-defective lentivirus carrying GFP (LV-GFP)-labeled BM-MSCs under a common microscope; (B) In vitro LV-GFP-labeled BM-MSCs (magnification, $\mathrm{x} 20$ ); (C) in vivo exogenously administered BM-MSCs were mainly observed in the inflamed colon (magnification, $\mathrm{x} 20$ ).

the PBS-treated group compared with the BM-MSC-treated mice and the control group $(\mathrm{p}<0.05)$; however, there was no difference in Foxp3 mRNA expression between the BM-MSCtreated group and the control group.

\section{Discussion}

The use of animal models of mucosal inflammation in studies on the pathogenesis of IBD was first implemented almost half 


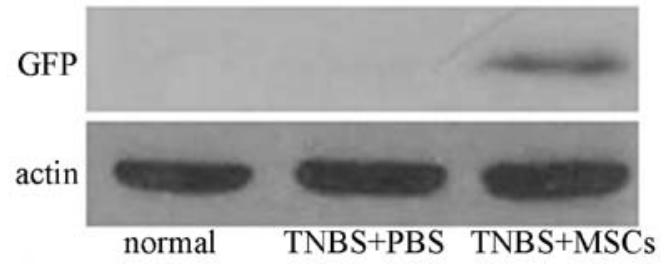

Figure 6. Western blot analysis of GFP protein. GFP protein was expressed in the group treated with GFP-labeled bone marrow-derived mesenchymal stem cells (BM-MSCs), but its expression was not observed in the PBS-treated group and the normal control.

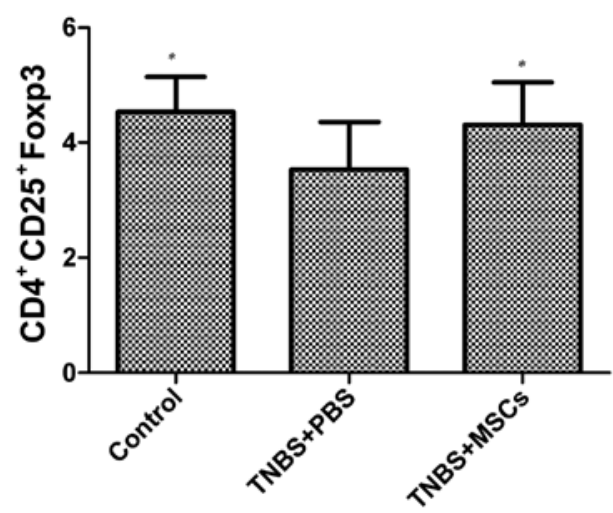

Figure 7. Effects of bone marrow-derived mesenchymal stem cells (BM-MSCs) on Tregs in peripheral blood. The proportion of Tregs was significantly decreased in the phosphate-buffered saline (PBS)-treated group compared with the control group $\left({ }^{*} \mathrm{p}<0.05\right)$. Following the administration of BM-MSCs, the number of Tregs increased compared with the PBS-treated group ( $(\mathrm{p}<0.05)$, and was equivalent to that of the control group.

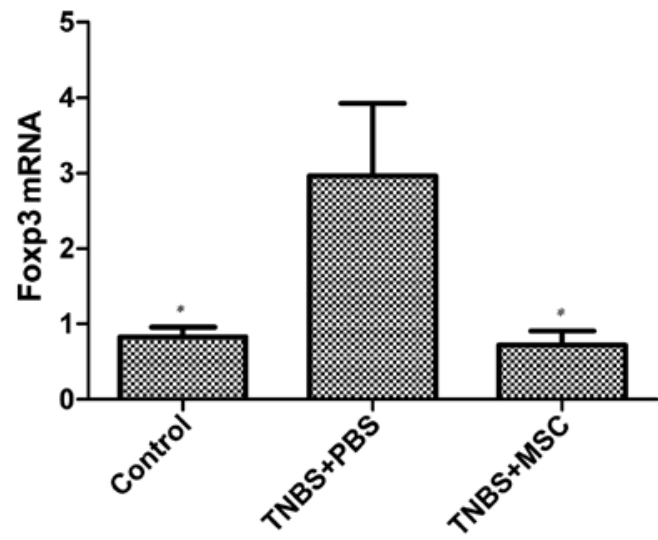

Figure 8. Colonic mucosas mRNA expression of Foxp3. An increased mucosal Foxp3 expression was observed in the phosphate-buffered saline (PBS)-treated group compared with the control group ( $\left.{ }^{*} \mathrm{p}<0.05\right)$. Following treatment with BM-MSCs, the mRNA expression of Foxp3 decreased significantly in the BM-MSC-treated group $(\mathrm{p}<0.05)$, and the levels were similar to the control group.

a century ago (25). Although animal models of IBD cannot represent the human disease completely, these types of studies are valuable and constitute serious attempts at investigating the immunological basis of this type of disease. In our study, we used TNBS to induce a rat model of colitis.

The gut is an immunologically unique organ which is in direct contact with the external environment and must retain the ability to mount an adaptive response to commensal bacteria and food simultaneously in order to maintain tolerance (26). The gut immune system protects the host against pathological invasion and mediates the contact between the host and microbes. Failure of these regulatory pathways and imbalances of the microbiota can result in inflammatory processes, such as $\operatorname{IBD}(27,28)$. A number of specialized immune cells are involved in maintaining mucosal tolerance in the intestine. Foxp3 ${ }^{+}$Tregs are the most significant cells in modulating this interaction within the adaptive immune system. One of the first studies to describe an existence of a peripheral mechanism for the regulation of the immune system was published in the 1960s (29). In the late 1990s, subsequent in vitro and in vivo studies revealed the immunosuppressive properties of Tregs $(30,31)$; however, the mechanisms involved are complex and multifactorial. A few years later, Fontenot et al (11) suggested that the Foxp3 gene, which encodes the forkhead family transcription factor, Foxp3, is the master regulatory gene for the development and function of Tregs. In clinical practice, there is a severe autoimmune syndrome termed immune dysregulation, polvendocrinopathy, enteropathy, X-linked (IPEX), characterized by a dysfunction in the development of Tregs and the consequent activation of autoreactive $\mathrm{T}$ cells which is caused by mutations within the Foxp3 gene in male infants $(10,32)$. In addition to IPEX, the most compelling evidence of the role of Tregs in IBD has come from the $T$ cell transfer model of colitis. In this model, naive $\mathrm{CD}^{+} \mathrm{T}$ cells with depleted Tregs are adoptively transferred into mice lacking $\mathrm{B}$ and $\mathrm{T}$ lymphocytes. In the intestine, these $T$ cells proliferate and become activated in response to bacterial antigens, resulting in inflammation and colitis. Thus, disease can be both induced and treated through the elimination of the microbiota or the cotransfer of Tregs $(33,34)$.

Interest in MSCs for their application in transplantation was fostered not only due to their ability to differentiate into different lineages, but also due to their capacity to suppress the immune response (35). In recent years, the potential application of both MSCs and Tregs in the treatment of a number of chronic inflammatory and autoimmune diseases has gained significant interest from immunologists worldwide, and the induction of Tregs by activated MSCs is now a wellpublicized phenomenon. In transplantation, one of the first studies which demonstrated that following the administration of MSCs there was an in vivo induction of Tregs, was the study by Casiraghi et al (36). In a subsequent study, pre-transplant administration of donor-derived MSCs into the portal vein in a semi-allogeneic heart transplant mouse model resulted in $\mathrm{T}$ cell hyporesponsiveness, prolonged cardiac allograft survival and expanded donor-specific Tregs expressing CD4, $\mathrm{CD} 25$ and Foxp3. Further evidence that the production of Tregs by MSCs resulting from graft survival was provided by a kidney allograft mouse model. It was also demonstrated that there was a significant increase in the number of intragraft Foxp $3^{+}$cells following treatment with MSCs, through which it was suggested that Tregs could recruit to the renal allograft (37).

In this study, we demonstrate that BM-MSCs can ameliorate TNBS-induced colitis in rats. To the best of our knowledge, this study is the first to demonstrate that BM-MSC therapy affects Foxp $3^{+}$Treg cells in the blood and gut mucosa, enhancing the treatment effects in IBD. After the rats with 
colitis received exogenously infused BM-MSCs, we assessed stool, body weight, as well as histological injury scores. Body weight and a marked histological improvement was noted in the MSC-treated group compared with the PBS-treated group. We hypothesize that the beneficial effects of treatment with MSCs may be linked to the induction of the activity of Tregs. Tregs in peripheral blood were assayed by flow cytometry and the mRNA expression of Foxp3 in the colon was determined by RT-PCR. Our data demonstrated that prior to the administration of BM-MSCs, the rats with IBD had lower levels of Foxp $3^{+}$cells in the peripheral blood compared with the healthy controls $(\mathrm{p}<0.05)$ (Fig. 7), but had higher mRNA levels of Foxp3 in the intestinal mucosa compared with the healthy controls $(p<0.05)$ (Fig. 8). The decrease in the circulating population in the peripheral blood may be due to an active pooling to the intestine and it is impossible to repopulate the blood without ample numbers of new Tregs. Our results on the expression of Foxp3 were similar to those from a recent study (38). Following the administration of BM-MSCs, the number of Foxp $3^{+}$cells in the peripheral blood increased compared with the PBS-treated group (p<0.05) (Fig. 7), but was lower compared with the healthy controls. In the intestinal mucosa, a decreased mucosal Foxp3 expression was observed in the BM-MSC-treated group compared with the PBS-treated group ( $\mathrm{p}<0.05)$ (Fig. 8); there was no significant difference between the control group and the MSC-treated group. In conclusion, there was an inverse regulation of mucosal and peripheral Foxp3 expression, which suggests that MSCs redistributed the Tregs from the mucosa to the blood.

The more we understand the complex interactions between MSCs and Tregs, the better we are likely to expore new treatments for a number of immune diseases. However, a number of studies have demonstrated that administered animal-derived MSCs can enhance tumor growth in certain experimental models (39). Beyond that, there are factors, such as the timing of delivery, the number of cells delivered, and the site of MSC infusion, which may affect the engraftment efficiency and the destination of exogenously delivered cells (40). Therefore, the possible long-term adverse effects and the details required to optimize the protocol for MSC delivery require further and thorough investigation. In conclusion, the interactions between transplantated bone marrow MSCs and Tregs should be further investigated. MSCs have tremendous potential for use in the treatment of IBD.

\section{Acknowledgements}

Financial support for this study was provided by a grant from the National Natural Science Foundation of China (no. 81102690)

\section{References}

1. Baumgart DC and Carding SR: Inflammatory bowel disease: cause and immunobiology. Lancet 369: 1627-1640, 2007.

2. Bouma $\mathrm{G}$ and Strober W: The immunological and genetic basis of inflammatory bowel disease. Nat Rev Immunol 3: 521-533, 2003.

3. Xavier RJ and Podolsky DK: Unravelling the pathogenesis of inflammatory bowel disease. Nature 448: 427-434, 2007.

4. Baumgart DC and Sandborn WJ: Inflammatory bowel disease: clinical aspects and established and evolving therapies. Lancet 369: 1641-1657, 2007.
5. Himmel ME, Hardenberg G, Piccirillo CA, et al: The role of T-regulatory cells and Toll-like receptors in the pathogenesis of human inflammatory bowel disease. Immunology 125: 145-153, 2008.

6. Fiocchi C: Towards a 'cure' for IBD. Dig Dis 30: 428-433, 2012.

7. Shevach EM: Mechanisms of foxp $3^{+} \mathrm{T}$ regulatory cell-mediated suppression. Immunity 30: 636-645, 2009.

8. Wing K and Sakaguchi S: Regulatory T cells exert checks and balances on self tolerance and autoimmunity. Nat Immunol 11: 7-13, 2010.

9. Bacchetta R, Passerini L, Gambineri E, et al: Defective regulatory and effector $\mathrm{T}$ cell functions in patients with FOXP3 mutations. J Clin Invest 116: 1713-1722, 2006.

10. McMurchy AN, Di Nunzio S, Roncarolo MG, et al: Molecular regulation of cellular immunity by FOXP3. Adv Exp Med Biol 665: 30-46, 2009.

11. Fontenot JD, Gavin MA and Rudensky AY: Foxp3 programs the development and function of $\mathrm{CD} 4{ }^{+} \mathrm{CD} 25^{+}$regulatory T cells. Nat Immunol 4: 330-336, 2003.

12. Mottet C, Uhlig HH and Powrie F: Cutting edge: cure of colitis by $\mathrm{CD}^{+} \mathrm{CD} 25^{+}$regulatory T cells. J Immunol 170: 3939-3943, 2003.

13. Yi T and Song SU: Immunomodulatory properties of mesenchymal stem cells and their therapeutic applications. Arch Pharm Res 35: 213-221, 2012.

14. Potian JA, Aviv H, Ponzio NM, et al: Veto-like activity of mesenchymal stem cells: functional discrimination between cellular responses to alloantigens and recall antigens. J Immunol 171: 3426-3434, 2003.

15. Le Blanc K, Tammik C, Rosendahl K, et al: HLA expression and immunologic properties of differentiated and undifferentiated mesenchymal stem cells. Exp Hematol 31: 890-896, 2003.

16. Engela AU, Baan CC, Dor FJ, et al: On the interactions between mesenchymal stem cells and regulatory $\mathrm{T}$ cells for immunomodulation in transplantation. Front Immunol 3: 126, 2012.

17. Le Blanc K, Frassoni F, Ball L, et al: Mesenchymal stem cells for treatment of steroid-resistant, severe, acute graft-versus-host disease: a phase II study. Lancet 371: 1579-1586, 2008.

18. Dalal J, Gandy K and Domen J: Role of mesenchymal stem cell therapy in Crohn's disease. Pediatr Res 71: 445-451, 2012.

19. Sun S, Guo Z, Xiao X, et al: Isolation of mouse marrow mesenchymal progenitors by a novel and reliable method. Stem Cells 21: 527-535, 2003.

20. Morris GP, Beck PL, Herridge MS, et al: Hapten-induced model of chronic inflammation and ulceration in the rat colon. Gastroenterology 96: 795-803, 1989.

21. Vowinkel T, Kalogeris TJ, Mori M, et al: Impact of dextran sulfate sodium load on the severity of inflammation in experimental colitis. Dig Dis Sci 49: 556-564, 2004.

22. González R, Rodríguez S, Romay C, et al: Anti-inflammatory activity of phycocyanin extract in acetic acid-induced colitis in rats. Parmacol Res 39: 55-59, 1999.

23. Powrie F, Correaoliveira R, Mauze S, et al: Regulatory interactions between $\mathrm{Cd} 45 \mathrm{Rb}$ (high) and $\mathrm{Cd} 45 \mathrm{Rb}$ (low) $\mathrm{Cd} 4(+) \mathrm{T}$-cells are important for the balance between protective and pathogenic cell-mediated immunity. J Exp Med 179: 589-600, 1994.

24. Read S, Malmstrom V and Powrie F: Cytotoxic T lymphocyteassociated antigen 4 plays an essential role in the function of CD25(+)CD4(+) regulatory cells that control intestinal inflammation. J Exp Med 192: 295-302, 2000.

25. Strober W: Animal models of inflammatory bowel disease - an overview. Dig Dis Sci 30: 3S-10S, 1985.

26. Neuman MG.Signaling forinflammation and repair in inflammatory bowel disease. Rom J Gastroenterol 13: 309-316, 2004.

27. Reiff C and Kelly D: Inflammatory bowel disease, gut bacteria and probiotic therapy. Int J Med Microbiol 300: 25-33, 2010.

28. Cho JH: The genetics and immunopathogenesis of inflammatory bowel disease. Nat Rev Immunol 8: 458-466, 2008.

29. Chaperon EA, Selner JC and Claman HN: Migration of antibody-forming cells and antigen-sensitive precursors between spleen, thymus and bone marrow. Immunology 14: 553-561, 1968.

30. Sakaguchi S, Sakaguchi N, Asano M, et al: Immunologic self-tolerance maintained by activated $\mathrm{T}$ cells expressing IL-2 receptor alpha-chains (CD25). Breakdown of a single mechanism of self-tolerance causes various autoimmune diseases. J Immunol 155: 1151-1164, 1995.

31. Takahashi T, Kuniyasu Y, Toda M, Sakaguchi N, Itoh M, Iwata M, et al: Immunologic self-tolerance maintained by $\mathrm{CD} 25^{+} \mathrm{CD} 4^{+}$naturally anergic and suppressive T cells: induction of autoimmune disease by breaking their anergic/suppressive state. Int Immunol 10: 1969-1980, 1998. 
32. d'Hennezel E, Bin Dhuban K, Torgerson T, et al: The immunogenetics of immune dysregulation, polyendocrinopathy, enteropathy, X linked (IPEX) syndrome. J Med Genet 49: 291-302, 2012.

33. Maloy KJ: Induction and regulation of inflammatory bowel disease in immunodeficient mice by distinct $\mathrm{CD} 4^{+} \mathrm{T}$-cell subsets. Methods Mol Biol 380: 327-335, 2007.

34. Eri R, McGuckin MA and Wadley R: T cell transfer model of colitis : a great tool to assess the contribution of T cells in chronic intestinal inflammation. Methods Mol Biol 844: 261-275, 2012.

35. Tolar, J, Le Blanc K, Keating A and Blazar BR: Concise review: hitting the right spot with mesenchymal stromal cells. Stem Cells 28: 1446-1455, 2010.

36. Casiraghi F, Azzollini N, Cassis P, et al: Pretransplant infusion of mesenchymal stem cells prolongs the survival of a semiallogeneic heart transplant through the generation of regulatory T cells. J Immunol 181: 3933-3946, 2008.
37. Ge W, Jiang J, Arp J, et al: Regulatory T-cell generation and kidney allograft tolerance induced by mesenchymal stem cells associated with indoleamine 2,3-dioxygenase expression. Transplantation 90: 1312-1320, 2010.

38. Li Z, Arijs I, De Hertogh G, et al: Reciprocal changes of Foxp3 expression in blood and intestinal mucosa in IBD patients responding to infliximab. Inflamm Bowel Dis 16: 1299-1310, 2010.

39. Djouad F, Plence P, Bony C, et al: Immunosuppressive effect of mesenchymal stem cells favors tumor growth in allogeneic animals. Blood 102: 3837-3844, 2003.

40. Karp JM and Leng Teo GS: Mesenchymal stem cell homing: the devil is in the details. Cell Stem Cell 4: 206-216, 2009. 